\title{
ON THE SINGULAR COMPONENTS OF A COPULA
}

\author{
FABRIZIO DURANTE, ${ }^{*}$ Free University of Bozen-Bolzano \\ JUAN FERNÁNDEZ-SÁNCHEZ,** Universidad de Almería \\ WOLFGANG TRUTSCHNIG, ${ }^{* * *}$ University of Salzburg
}

\begin{abstract}
We analyze copulas with a nontrivial singular component by using their Markov kernel representation. In particular, we provide existence results for copulas with a prescribed singular component. The constructions not only help to deal with problems related to multivariate stochastic systems of lifetimes when joint defaults can occur with a nonzero probability, but even provide a copula maximizing the probability of joint default.
\end{abstract}

Keywords: Copula; coupling; disintegration theorem; Markov kernel; singular measure 2010 Mathematics Subject Classification: Primary 60E05

Secondary 28A50; $91 \mathrm{G} 70$

\section{Introduction}

Copula models have become popular in different applications in view of their ability to describe a variety of different relationships among random variables [7], [8]. Such a growing interest also motivates the introduction of methodologies that go beyond classical statistical assumptions, such as absolute continuity or smoothness of derivatives (i.e. conditional distribution functions), that sometimes may impose undesirable constraints on the underlying stochastic model.

In order to clarify the concepts, consider the case when copulas are employed to describe the dependence among two (or more) lifetimes, i.e. positive random variables. Such models arise, at least, in two different domains. In engineering applications, joint models of lifetimes may be related to a system composed of several components that operate until only some of their components work while the others have already failed [4], [15], [16]. In portfolio credit risk modeling, however, the lifetimes have the interpretation of default times (of firms, or obligors), while their copula serves to model the possible effect of one asset default on the probability of default of the other assets, which would require special care to avoid underestimation of the risk of the portfolio; see, e.g. [13]. In both cases, it is important to estimate the probability of the occurrence of a joint default, which means, in the case of a bivariate random vector $(X, Y)$, the probability of the event $\{X=Y\}$. Now, as stressed, for instance, in [14], if one requires that the event $\{X=Y\}$ has nonzero probability, then it would be important to select a copula for $(X, Y)$ that has a singular component (i.e. it cannot be described via a single density function) and, in addition, its singular component spreads at least a part of its probability mass along a

\footnotetext{
Received 13 June 2014; revision received 7 October 2014.

* Postal address: Faculty of Economics and Management, Free University of Bozen-Bolzano, Bolzano, Italy. Email address: fabrizio.durante@unibz.it

** Postal address: Grupo de Investigación de Análisis Matemático, Universidad de Almería, La Cañada de San Urbano, Almería, Spain. Email address: juan.fernandez@ual.es

*** Postal address: Department for Mathematics, University of Salzburg, Salzburg, Austria.

Email address: wolfgang@trutschnig.net
} 
set determined by the marginals $F_{X}$ and $F_{Y}$ given by $\left\{(u, v) \in[0,1]^{2}: F_{X}^{-1}(u)=F_{Y}^{-1}(v)\right\}$, when $F_{X}$ and $F_{Y}$ are strictly increasing.

Motivated by these applications, it is hence desirable to provide a theoretical background for the study of copulas with singular components. In fact, as stressed, for instance, in [3], the related literature presents several statements that are not sound unless further assumptions are required. Here, we aim at clarifying several aspects concerning copulas with singular components. We provide examples and counterexamples that are of pathological nature, but may serve to warn against any simplistic thinking about the dependence structure. Finally, we present some existence results showing how to obtain copulas assigning positive mass to the graph of a function.

\section{Basic definitions and properties of singular copulas}

First of all, we recall some basic definitions about copulas. Any two-dimensional copula $C$ is a multivariate distribution function on $[0,1]^{2}$ whose univariate margins are uniformly distributed on $[0,1]$. As such, it induces a probability measure $\mu_{C}$ on the Borel sets $B\left([0,1]^{2}\right)$ of $[0,1]^{2}$. Moreover, since $\mu_{C}$ cannot have atoms, in view of the Lebesgue decomposition theorem (see, e.g. [1, Theorem 2.2.6]), we have

$$
\mu_{C}=\mu_{C}^{\mathrm{a}}+\mu_{C}^{\mathrm{s}}
$$

where:

- $\mu_{C}^{\mathrm{a}}$ is a measure on $\mathscr{B}\left([0,1]^{2}\right)$ that is absolutely continuous with respect to the $d$-dimensional Lebesgue measure $\lambda_{2}$;

- $\mu_{C}^{\mathrm{s}}$ is a measure on $\mathcal{B}\left([0,1]^{2}\right)$ that is singular with respect to the Lebesgue measure on $[0,1]^{2}$, i.e. the probability measure is concentrated on a set $B$ such that $\lambda_{2}(B)=0$.

If $\mu_{C}^{\mathrm{s}}\left([0,1]^{2}\right)=1-\alpha>0$, then $C$ has a singular component of total mass equal to $1-\alpha$.

The support of the singular component of a copula $C$ is the complement of the union of all open subsets of $[0,1]^{d}$ with $\mu_{C}^{\mathrm{s}}$-measure 0 . In other words, it is the smallest closed set on which $\mu_{C}^{\mathrm{s}}$ is concentrated. It is worth noting that this support needs not be concentrated on graphs of functions (as in the case of Marshall-Olkin copulas) or segments (as for shuffles of Min-[5,19]). In fact, using the results in [2] and [6], for any $s \in[1,2]$, there exists a singular copula whose support has Hausdorff dimension $s$, as well as singular copulas with full support whose conditional distribution functions are continuous, strictly increasing, and have derivative 0 almost everywhere (a.e.); see [19]. Therefore, up to simple cases, the mass of the singular component cannot be identified by looking at regions where the conditional distribution functions related to the copula (i.e. the derivatives) have jumps (cf. [9, pp. 14-16] and [10, Theorem 8.1]). In the following section, we clarify these aspects by using the notion of a Markov kernel.

\section{Singular copulas versus singular Markov kernels}

The symbol $\mathcal{P}\left([0,1]^{2}\right)$ will denote the family of all probability measures on $[0,1]^{2}$. If $\mathbb{P}^{(X, Y)} \in \mathcal{P}\left([0,1]^{2}\right)$, then $\mathbb{P}^{X}$ (respectively, $\mathbb{P}^{Y}$ ) denotes the marginal law defined on every Borel set $E \subset[0,1]$ by $\mathbb{P}^{X}(E)=\mathbb{P}^{(X, Y)}(E \times[0,1])$ (respectively, $\mathbb{P}^{Y}(E)=\mathbb{P}^{(X, Y)}([0,1] \times E)$ ). Let $\mathcal{C}$ denote the family of all two-dimensional copulas. For regular conditional distributions, Markov kernels and disintegration theorems, we refer the reader to [11]. Moreover, a mapping $K:[0,1] \times \mathscr{B}([0,1]) \rightarrow[0,1]$ will be called substochastic kernel if $x \mapsto K(x, E)$ is Borel 
measurable in $x$ for every $E \in \mathscr{B}([0,1])$ and $E \mapsto K(x, E)$ is a measure of total mass less than or equal to 1 for every $x \in[0,1]$. If $K(x, \cdot)$ is a probability measure on $[0,1]$, then $K$ will be called a Markov kernel; see, for example, [12].

Given $A \in \mathcal{C}$ with $(X, Y) \sim A$, we denote (a version of) the regular conditional distribution of $Y$ given $X$ by $K_{A}(\cdot, \cdot)$ and refer to it as a Markov kernel of $A$. It follows that, for every Borel set $F \in \mathscr{B}([0,1])$, we have (with $F_{x}=\{y \in[0,1]:(x, y) \in F\}$ )

$$
\int_{[0,1]} K_{A}\left(x, F_{x}\right) \mathrm{d} \lambda(x)=\mu_{A}(F) .
$$

We start with the following simple observation.

Lemma 1. Suppose that a copula $C$ has a singular component, i.e. $\mu_{C}^{\mathrm{s}}\left([0,1]^{2}\right)=1-\alpha>0$. If $(X, Y) \sim \mathbb{P}^{(X, Y)}=(1 /(1-\alpha)) \mu_{C}^{\mathrm{s}} \in \mathcal{P}\left([0,1]^{2}\right)$, then the marginal laws $\mathbb{P}^{X}$ and $\mathbb{P}^{Y}$ are absolutely continuous. If $g_{X}$ denotes the density of $\mathbb{P}^{X}$ and $K_{C}^{\mathrm{s}}$ the Markov kernel of $(1 /(1-\alpha)) \mu_{C}^{\mathrm{s}}$, then

$$
\frac{1}{1-\alpha} \mu_{C}^{\mathrm{s}}(E \times F)=\mathbb{P}(X \in E, Y \in F)=\int_{[0,1]} K_{C}^{\mathrm{s}}(x, F) g_{X}(x) \mathrm{d} \lambda(x)
$$

as well as $(1-\alpha) K_{C}^{\mathrm{s}}(x,[0,1]) g_{X}(x) \leq 1$ for $\lambda$-almost every $x \in[0,1]$.

Proof. Let $E \in \mathscr{B}([0,1])$, then we have

$$
\mathbb{P}^{X}(E)=\frac{1}{1-\alpha} \mu_{C}^{\mathrm{s}}(E \times[0,1]) \leq \frac{1}{1-\alpha} \mu_{C}(E \times[0,1])=\frac{1}{1-\alpha} \lambda(E)
$$

so $X$ is absolutely continuous (analogously, $Y$ is absolutely continuous). The remaining part of the proof is a direct consequence of the disintegration theorem.

Thanks to the previous lemma, we can prove that the singularity of a copula depends on the singularity of the related Markov kernels, as stated in the following result.

Theorem 1. Suppose that $C \in \mathcal{C}$. Then the following two conditions are equivalent:

(i) $C$ is singular;

(ii) there exists a set $\Lambda \in \mathscr{B}([0,1])$ with $\lambda(\Lambda)=1$ such that the measure $K_{C}(x, \cdot)$ is singular with respect to $\lambda$ for every $x \in \Lambda$.

Proof. Note that (i) implies (ii). If $C$ is singular then, by definition, there exists a set $N \in \mathscr{B}\left([0,1]^{2}\right)$ with $\mu_{C}(N)=1$ and $\lambda_{2}(N)=0$. Applying disintegration to $\mu_{C}$ and $\lambda_{2}$ directly yields the existence of $\Lambda \in \mathscr{B}([0,1])$ such that $\lambda\left(N_{x}\right)=0$ and $K_{C}\left(x, N_{x}\right)=1$ for every $x \in \Lambda$ whereby $N_{x}=\{y \in[0,1]:(x, y) \in N\}$ denotes the $x$-cut of $N$.

Note that (ii) implies (i). Suppose that $C$ is not fully singular, i.e. $\mu_{C}^{\mathrm{a}}\left([0,1]^{2}\right)=: \alpha>0$. Let $k$ denote the density of $\mu_{C}^{\mathrm{a}}$. Without loss of generality, we may assume that $\int_{[0,1]} k(x, y) \mathrm{d} \lambda(y) \leq$ 1 for every $x \in[0,1]$. If $\alpha=1$, then the result follows immediately; so we examine the case when $\alpha \in(0,1)$. Letting $K_{C}^{\mathrm{s}}$ denote the Markov kernel of $(1 /(1-\alpha)) \mu_{C}^{\mathrm{s}}$ and using Lemma 1 , it follows that

$$
\mu_{C}(E)=(1-\alpha) \int_{[0,1]} K_{C}^{\mathrm{s}}\left(x, E_{x}\right) g(x) \mathrm{d} \lambda(x)+\int_{[0,1]} \int_{E_{x}} k(x, y) \mathrm{d} \lambda(y) \mathrm{d} \lambda(x)
$$


for every $E \in \mathcal{B}\left([0,1]^{2}\right)$. Define

$$
K(x, F)=(1-\alpha) K_{C}^{\mathrm{s}}(x, F) g(x)+\int_{F} k(x, y) \mathrm{d} \lambda(y)
$$

for every $x \in[0,1]$ and $F \in \mathscr{B}([0,1])$, then $K(x, \cdot)$ is a finite measure on $\mathscr{B}([0,1])$ and a measurable function in $x$ for fixed $F$. Using (1) it is straightforward to verify that $K(x,[0,1])=$ 1 for $\lambda$-a.e. $x \in[0,1]$ so redefining $K(x, \cdot)$ (at most) on a set of measure 0 yields a Markov kernel $K_{C}$ of $C$. Since $\int_{[0,1]^{2}} k \mathrm{~d} \lambda_{2}=\alpha>0$ we cannot have $(1-\alpha) K_{C}^{\mathrm{s}}(x,[0,1]) g(x)=1$ for $\lambda$-a.e. $x \in[0,1]$.

As a direct application of the results in [12] the Markov kernel $K_{C}$ of an arbitrary copula $C \in \mathcal{C}$ can be decomposed into the sum of three substochastic kernels $K_{C}^{\mathrm{a}}, K_{C}^{\mathrm{s}}, K_{C}^{\mathrm{d}}$, i.e.

$$
K_{C}(x, E)=K_{C}^{\mathrm{a}}(x, E)+K_{C}^{\mathrm{s}}(x, E)+K_{C}^{\mathrm{d}}(x, E)
$$

for every $x \in[0,1]$ and $E \in \mathscr{B}([0,1])$. Thereby, the measure $K_{C}^{\mathrm{a}}(x, \cdot)$ is absolutely continuous with respect to $\lambda$, the measure $K_{C}^{\mathrm{s}}(x, \cdot)$ is singular with respect to $\lambda$ and has no point masses, and $K_{C}^{\mathrm{d}}(x, \cdot)$ is discrete for every $x \in[0,1]$.

Examples of copulas whose Markov kernels show all three characteristics above are easily constructed. Consider the copula $A=\frac{1}{3}\left(\Pi_{2}+S+T\right)$, whereby $\Pi_{2}$ is the independence copula, $S$ is one of the very singular copulas with full support from [19], and $T$ is the singular copula with full support from [3]. Note that all three copulas that appear in the expression of $A$ have full support $[0,1]^{2}$.

\section{The singular mass of a copula and copulas whose singular mass is concentrated on the graphs of functions}

In the previous section we investigated the main properties of the copulas with singular component with respect to the Markov kernel representation. Now, we would like to raise the question as to whether we may use such a representation in order to provide examples of copulas with a prescribed distribution of the singular component.

First, suppose that $C$ is a copula, $K_{C}$ is its Markov kernel, and that $K_{C}$ is decomposed according to (2). Set

$$
a_{C}(x):=K_{C}^{\mathrm{d}}(x,[0,1])
$$

for every $x \in[0,1]$ (note that $a_{C}$ depends on the concrete choice of the kernel and is therefore only defined uniquely $\lambda$-a.e.). Then $a_{C}$ is measurable and we can (without unnecessary additional assumptions) calculate $\int_{[0,1]} a_{C} \mathrm{~d} \lambda$, i.e. the way in which the discrete component spreads its mass. It turns out that, in general, the function $a_{C}$ can be very irregular, as the following result shows (cf. [9, p. 15]).

Theorem 2. For every measurable function $f:[0,1] \rightarrow[0,1]$ there exists a copula $C$ such that $a_{C}=f$.

Proof. Let $f$ be a measurable function such that $\|f\|_{1}=\ell$. If $\ell \in\{0,1\}$, then the result easily follows by considering the independence copula and the comonotonicity copula, respectively. Hence, assume that $\|f\|_{1} \in(0,1)$. We will construct a copula $C$ with $a_{C}=f$ such that the singular component of $C$ is concentrated on the diagonal $\Delta=\{(x, x): x \in[0,1]\}$ of the unit square. The procedure is as follows. Let $\mu$ denote the measure on $\mathscr{B}\left([0,1]^{2}\right)$ fulfilling 
$\mu(\Delta)=\mu\left([0,1]^{2}\right)$ as well as $\mu\left([0, x]^{2}\right)=\int_{[0, x]} f \mathrm{~d} \lambda$ for every $x \in[0,1]$. Let $S(x, y):=$ $\mu([0, x] \times[0, y])$ the corresponding measure-generating function. Then $S$ is symmetric and its marginal $F:[0,1] \rightarrow[0,1]$, given by $F(x)=S(x, 1)=\int_{[0, x]} f \mathrm{~d} \lambda$, is Lipschitz continuous (with Lipschitz constant 1), and fulfills $F(1)=\|f\|_{1} \in(0,1)$. Setting $G:[0,1] \rightarrow[0,1]$ by $G(x)=x-F(x)$, we have therefore another Lipschitz continuous measure-generating function fulfilling $G(0)=0$ and $G(1) \in(0,1)$. Define $T$ and $\vartheta$ by

$$
T(x, y):=\vartheta([0, x] \times[0, y]):=\frac{1}{G(1)} G(x) G(y)
$$

for all $x, y \in[0,1]$. Note that such a $T$ is obtained as a product of its univariate marginals (so, it is constructed via the independence copula); however, any other absolutely continuous copula can be used as well. It follows that $\vartheta$ is absolutely continuous with respect to $\lambda_{2}$. Finally, considering

$$
C(x, y)=S(x, y)+T(x, y)
$$

it follows immediately that $C$ is a copula whose singular component is $\mu$. Applying disintegration we directly obtain $a_{C}=f \lambda$-a.e. Since the Markov kernel of a copula is only defined uniquely a.e., the proof is completed.

Using essentially the same idea of the previous proof, we can show that for every transformation $T:[0,1] \rightarrow[0,1]$ being nonsingular (i.e. the push-forward $\lambda^{T}$ is absolutely continuous with respect to $\lambda$ ), we find a copula $A$ such that the corresponding kernel $K_{A}$ fulfills $K_{A}(x,\{T x\})>0$ for every $x \in[0,1]$. In other words, the singular components of copulas can be concentrated on the graphs of arbitrary nonsingular transformations.

Theorem 3. For every nonsingular transformation $T:[0,1] \rightarrow[0,1]$ there exists a copula $A$ such that the singular component of $A$ is concentrated on the graph $\Gamma(T)$ of $T$. Moreover, $K_{A}(x,\{T x\})>0$ for every $x \in[0,1]$.

Proof. Suppose that $T:[0,1] \rightarrow[0,1]$ is nonsingular and let $f$ denote the density of $\lambda^{T}$ with respect to $\lambda$. Define $a:[0,1] \rightarrow[0,1]$ by

$$
a(x)= \begin{cases}1 & \text { if } f \circ T(x) \leq 1, \\ \frac{1}{f \circ T(x)} & \text { if } f \circ T(x)>1 .\end{cases}
$$

Using the Cauchy-Schwarz inequality it is straightforward to verify that $\int_{[0,1]} a \mathrm{~d} \lambda=1$ if and only if $T$ is $\lambda$-preserving. Since for $\lambda$-preserving $T$ the (completely dependent) copula $A$ with the kernel $K_{A}(x, F)=\mathbf{1}_{F}(T x)$ has the properties stated in the theorem, we can focus on the $\int_{[0,1]} a \mathrm{~d} \lambda<1$ case. Let $\delta_{z}$ denote the Dirac measure in $z$ for every $z \in[0,1]$. Obviously, $K^{\mathrm{d}}:[0,1] \times \mathscr{B}([0,1]) \rightarrow[0,1]$, defined by

$$
K^{\mathrm{d}}(x, F):=a(x) \delta_{T x}(F)=a(x) \mathbf{1}_{F}(T x),
$$

is a substochastic kernel. Its induced measure $\mu$, given by

$$
\mu(E \times F)=\int_{E} a(x) \delta_{T x}(F) \mathrm{d} \lambda(x),
$$

fulfills $\mu\left([0,1]^{2}\right)=\int_{[0,1]} a \mathrm{~d} \lambda \in(0,1)$. Let $S$ denote the corresponding measure-generating function and $F, G$ the measure-generating functions of the marginals $\mu^{\pi_{1}}$ and $\mu^{\pi_{2}}$. It is 
straightforward to verify that $F(1)=G(1)$. Moreover, $F$ has density $a$ and $G$ has density $g$ given by

$$
g(x)=f(x) \mathbf{1}_{[0,1]}(f(x))+\mathbf{1}_{(1, \infty)}(f(x)),
$$

i.e. both densities only take values in $[0,1]$. As a direct consequence $\hat{F}, \hat{G}:[0,1] \rightarrow[0,1]$, defined by

$$
\hat{F}(x)=\frac{x-F(x)}{1-F(1)}, \quad \hat{G}(y)=\frac{y-G(y)}{1-G(1)}
$$

are absolutely continuous distribution functions. Hence,

$$
R(x, y):=\frac{1}{1-F(1)}(x-F(x))(y-G(y))
$$

defines a two-dimensional measure-generating function. (Note that such an $R$ is obtained as a product of its univariate marginals; however, any other absolutely continuous copula can be used as well to construct it). Finally, it easily follows that $A_{T}:=S+R$ is a copula with the desired properties.

If we replace function $a$ in (4) by any other measurable function $b$ with $0<b(x) \leq a(x)$ $\lambda$-a.e. on $[0,1]$, we may obtain other copulas that concentrate mass on $\Gamma(T)$. Remarkably, the copula $A_{T}$ constructed in the proof of Theorem 3 does not only fulfill $K_{A}(x,\{T x\})$ for every $x>0$, but it even assigns a maximum possible mass to the graph $\Gamma(T)$.

Theorem 4. Let $T:[0,1] \rightarrow[0,1]$ be a nonsingular transformation and let $A_{T}$ denote the copula constructed in the proof of Theorem 3. Then we have $\sup _{B \in \mathcal{C}} \mu_{B}(\Gamma(T))=\mu_{A_{T}}(\Gamma(T))$.

Proof. As above, let $f$ denote the density of $\lambda^{T}$, let $a$ be defined according to (4), and set $F:=f^{-1}((1, \infty))$. Suppose that $B \in \mathcal{C}$ fulfills $\mu_{B}(\Gamma(T))>\mu_{A_{T}}(\Gamma(T)$ and set $b(x):=$ $K_{B}(x,\{T x\})$ for every $x \in[0,1]$. Since $b$ cannot be strictly greater than $a$ on $T^{-1}\left(F^{c}\right)$, it follows that $\int_{T^{-1}(F)} b \mathrm{~d} \lambda>\int_{T^{-1}(F)} a \mathrm{~d} \lambda$, from which we obtain

$$
\begin{aligned}
\lambda(F) & =\int_{[0,1]} K_{B}(x, F) \mathrm{d} \lambda(x) \\
& \geq \int_{[0,1]} b(x) \delta_{T x}(F) \mathrm{d} \lambda(x) \\
& =\int_{T^{-1}(F)} b \mathrm{~d} \lambda \\
& >\int_{T^{-1}(F)} a \mathrm{~d} \lambda \\
& =\int_{[0,1]} a(x) \delta_{T x}(F) \mathrm{d} \lambda(x) \\
& =\int_{[0,1]} \frac{1}{f \circ T(x)} \mathbf{1}_{F}(T(x)) \mathrm{d} \lambda(x) \\
& =\int_{[0,1]} \frac{1}{f(y)} \mathbf{1}_{F}(y) f(y) \mathrm{d} \lambda(y) \\
& =\lambda(F) .
\end{aligned}
$$

Hence, we cannot have $\mu_{B}(\Gamma(T))>\mu_{A_{T}}(\Gamma(T)$. 

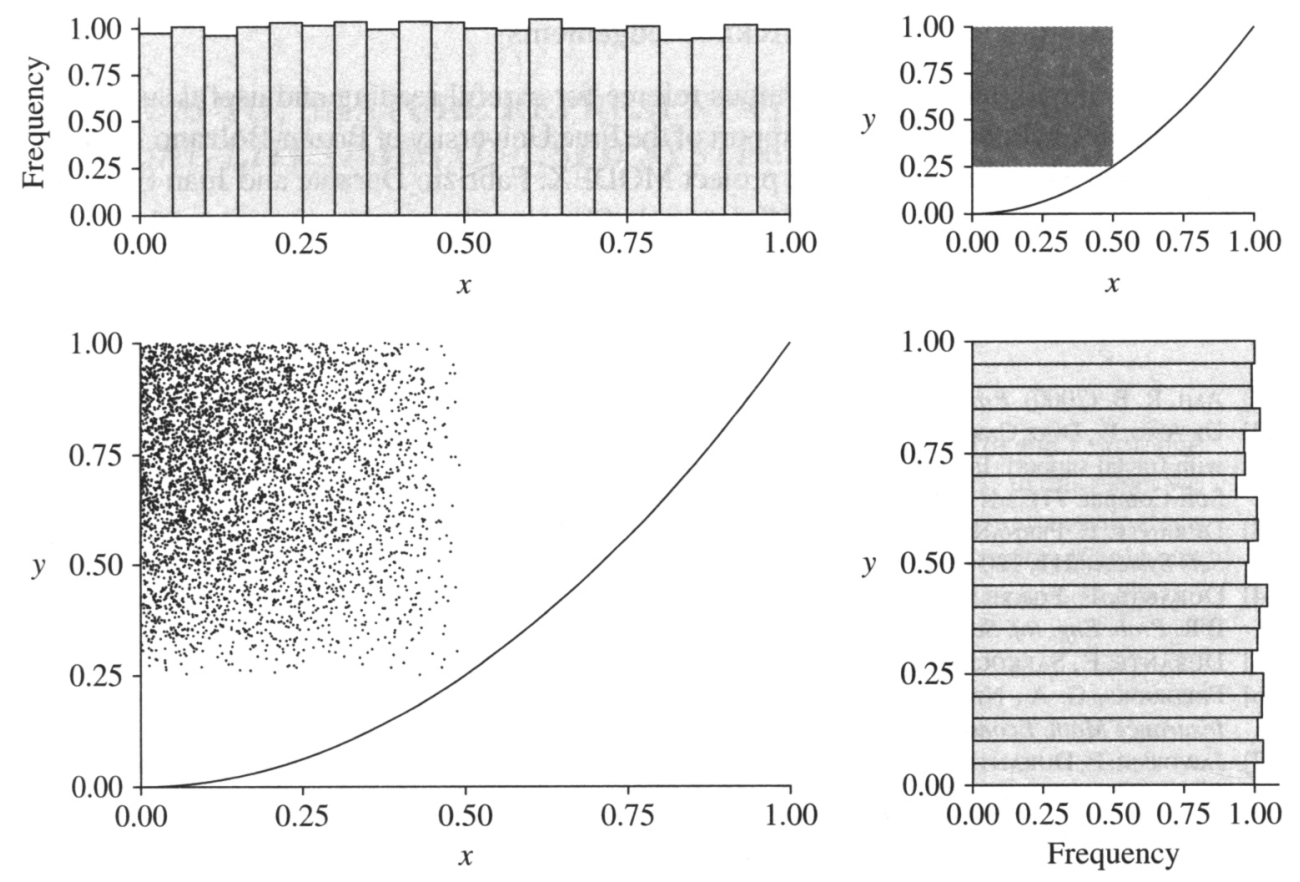

FiguRE 1: Scatterplot of a bivariate sample of size 20000 generated by the copula $A_{T}$ from Theorem 4 with $T(x)=x^{2}$ (lower left), including the marginal histograms as well as the support of the copula (upper right).

Theorem 3 implies the following result.

Corollary 1. Suppose that $T_{1}, T_{2}, T_{3}, \ldots:[0,1] \rightarrow[0,1]$ are nonsingular transformations. Then there exists a copula $A \in \mathcal{C}$ such that $K_{A}\left(x,\left\{T_{i} x\right\}\right)>0$ for every $x \in[0,1]$ and every $i \in \mathbb{N}$.

Proof. For every $i \in \mathbb{N}$ let $A_{i}:=A_{T_{i}}$ denote the copula according to Theorem 3 . Then the copula $B:=\sum_{i=1}^{\infty} \frac{1}{2^{i}} A_{i}$ has the stated property.

Finally, we discuss an interesting application. Following [14], suppose that $X, Y$ are two lifetimes with continuous and strictly increasing distribution functions $F_{X}$ and $F_{Y}$. Set $T(x):=$ $F_{Y} \circ F_{X}^{-1}(x)$. Then Theorem 4 ensures that the copula $A_{T}$ is such that $\mathbb{P}(X=Y)$ is maximal. In other words, a joint default is most likely to occur under this dependence structure. In Figure 1 we show a random sample from the copula $A_{T}$ when $T(x)=x^{2}$. Note that such problems are popular in the coupling literature, although they usually require additional assumptions such as the existence of marginal densities for $X$ and $Y$; see [17].

\section{Conclusions}

We have analyzed the class of copulas with a nontrivial singular component by using their Markov kernel representation. In particular, we provide existence results about copulas with prescribed singular component in a very general setting. We argue that such investigations may help to deal with copulas in many problems related to stochastic systems of lifetimes. 


\section{Acknowledgements}

We would like to thank the anonymous referee for careful reading and useful suggestions. Fabrizio Durante acknowledges the support of the Free University of Bozen-Bolzano, Faculty of Economics and Management, via the project MODEX. Fabrizio Durante and Juan FernándezSánchez have been supported by the Ministero de Ciencia e Innovación (Spain), under research project MTM2011-22394.

\section{References}

[1] Ash, R. B. (2000). Probability and Measure Theory, 2nd edn. Harcourt/Academic Press, Burlington, MA.

[2] De Amo, E., Díaz Carrillo, M. and Fernández-Sánchez, J. (2010). On concordance measures and copulas with fractal support. In Combining Soft Computing and Statistical Methods in Data Analysis (Adv. Intelligent Soft Comput. 77), eds C. Borgelt et al., Springer, Berlin, pp. 131-138.

[3] Durante, F., Fernández Sánchez, J. And Sempi, C. (2013). A note on the notion of singular copula. Fuzzy Sets Systems 211, 120-122.

[4] Durante, F., Foschi, R. And Spizzichino, F. (2010). Aging functions and multivariate notions of NBU and IFR. Prob. Eng. Inf. Sci. 24, 263-278.

[5] Durante, F., Sarkoci, P. ANd Sempi, C. (2009). Shuffles of copulas. J. Math. Anal. Appl. 352, 914-921.

[6] Fredricks, G. A., Nelsen, R. B. and RodríGuez-Lallena, J. A. (2005). Copulas with fractal supports. Insurance Math. Econom. 37, 42-48.

[7] JAWORSKI, P., DURANTE, F. AND HÄRdLE, W. K. (eds) (2013). Copulae in Mathematical and Quantitative Finance (Lecture Notes Statist. 213). Springer, Berlin.

[8] JaWorski, P., Durante, F., HÄrdle, W. K. AND Rychlik, T. (eds) (2010). Copula Theory and Its Applications (Lecture Notes Statist. 198). Springer, Berlin.

[9] JoE, H. (1997). Multivariate Models and Dependence Concepts (Monogr. Statist. Appl. Prob. 73). Chapman \& Hall, London.

[10] JoE, H. (2015). Dependence Modeling with Copulas. CRC, Boca Raton, FL.

[11] Kallenberg, O. (2002). Foundations of Modern Probability, 2nd edn. Springer, New York.

[12] Lange, K. (1973). Decompositions of substochastic transition functions. Proc. Amer. Math. Soc. 37, 575-580.

[13] MaI, J.-F. AND Scherer, M. (2012). Simulating Copulas (Ser. Quant. Finance 4). Imperial College Press, London.

[14] MaI, J.-F. AND Scherer, M. (2014). Simulating from the copula that generates the maximal probability for a joint default under given (inhomogeneous) marginals. In Topics in Statistical Simulation, eds V. B. Melas et al., Springer, New York, pp. 333-341.

[15] Navarro, J. ANd SpIzZichino, F. (2010). Comparisons of series and parallel systems with components sharing the same copula. Appl. Stoch. Models Business Industry 26, 775-791.

[16] Navarro, J., del Águila, Y., Sordo, M. A. ANd Suárez-Llorens, A. (2013). Stochastic ordering properties for systems with dependent identically distributed components. Appl. Stoch. Models Business Industry 29, 264-278.

[17] ThOrIsSON, H. (2000). Coupling, Stationarity, and Regeneration. Springer, New York.

[18] Trutschnig, W. And Fernández SÁnchez, J. (2013). Some results on shuffles of two-dimensional copulas. J. Statist. Planning Infer. 143, 251-260.

[19] Trutschnig, W. AND FERnáNDEZ-SÁNChEZ, J. (2014). Copulas with continuous, strictly increasing singular conditional distribution functions. J. Math. Anal. Appl. 410, 1014-1027. 